\title{
Universality in the Classical Limit of Massless Gravitational Scattering
}

\author{
Zvi Bern, ${ }^{1}$ Harald Ita๑, ${ }^{2}$ Julio Parra-Martinez $\odot,{ }^{1}$ and Michael S. Ruf ${ }^{2}$ \\ ${ }^{1}$ Mani L. Bhaumik Institute for Theoretical Physics, UCLA Department of Physics and Astronomy, Los Angeles, California 90095 , USA \\ ${ }^{2}$ Physikalisches Institut, Albert-Ludwigs-Universität Freiburg, Hermann-Herder-Strasse 3, 79104 Freiburg, Germany
}

(Received 13 February 2020; accepted 31 March 2020; published 17 July 2020)

\begin{abstract}
We demonstrate the universality of the gravitational classical deflection angle of massless particles through $\mathcal{O}\left(G^{3}\right)$ by studying the high-energy limit of full two-loop four-graviton scattering amplitudes in pure Einstein gravity as well as $\mathcal{N} \geq 4$ supergravity. As a by-product, our first-principles calculation provides a direct confirmation of the massless deflection angle in Einstein gravity proposed long ago by Amati, Ciafaloni, and Veneziano, and is inconsistent with a recently proposed alternative.
\end{abstract}

DOI: 10.1103/PhysRevLett.125.031601

Introduction.-The high-energy behavior of gravitational-scattering processes has a long and interesting history as a fundamental probe of gravitational theories at the classical and quantum level (see, e.g., Refs. [1-3]). The simplicity of scattering in the high-energy limit makes it a natural forum to extract information about high orders in perturbation theory. Indeed, using insight from string amplitudes and the analyticity of scattering amplitudes, Amati, Ciafaloni, and Veneziano (ACV) [3] worked out the high-energy limit of massless graviton scattering through $O\left(G^{3}\right)$ long before it became technically feasible to compute two-loop scattering amplitudes in quantum field theory directly. Using this they calculated the corresponding correction to the gravitational deflection angle of massless particles in general relativity.

Recently the subject of scattering processes in gravitational theories has been reinvigorated by the spectacular observation of gravitational waves by the LIGO and Virgo Collaborations [4]. While scattering processes may seem rather different from the bound-state problem of gravitational-wave generation, the underlying physics is the same. In particular, classical two-body potentials can be extracted from scattering processes [5], including new state-of-the-art calculations [6,7]. This approach leverages the huge advances in computing quantum scattering amplitudes that stem from the modern unitarity method [8] and from double-copy relations [9] between gauge and gravity theories.

The possibility of using quantum scattering amplitudes to obtain the classical deflection angle was also promoted by Damour [10], who used the ACV result for the conservative scattering angle to impose constraints on

Published by the American Physical Society under the terms of the Creative Commons Attribution 4.0 International license. Further distribution of this work must maintain attribution to the author(s) and the published article's title, journal citation, and DOI. Funded by SCOAP ${ }^{3}$. classical two-body Hamiltonians of the type used for gravitational-wave template construction [11]. In a very recent paper [12], however, Damour has cast doubt on the program of using quantum scattering amplitudes to extract information on classical dynamics. His central claim is that both the classical scattering angle derived by ACV and the $\mathcal{O}\left(G^{3}\right)$ two-body Hamiltonian derived in Refs. [6,7] are not correct. His claims, based on information obtained from the self-force (small mass ratio) expansion [13] of the boundstate dynamics as well as structural properties in the results of Ref. [7], provide results with a smooth transition between massive and massless classical scattering.

In this Letter we confirm that the conservative scattering angle as determined by ACV [3] is indeed correct. Our confirmation follows as a by-product of studying universality of the classical scattering angle in massless theories. Remarkably, we find that the scattering angle through $\mathcal{O}\left(G^{3}\right)$ is independent of the matter content for a variety of theories, implying graviton dominance in the high-energy limit. This dominance is well known at leading eikonal order [2]. Reference [14] revealed early hints of such dominance via analysis of gravitino contributions at the next nonvanishing order.

Our study relies on having on hand the explicit expressions for massless two-loop four-point amplitudes for $\mathcal{N} \geq$ 4 supergravity [15-17] and pure Einstein gravity [18]. The latter result makes use of the latest advances in evaluating multiloop amplitudes based on numerical unitarity followed by analytic reconstructions [19]. Armed with the fully evaluated amplitudes we then follow the standard [20] and widely used (see, e.g., Refs. [21-25]) extractions of the scattering angle, using both impact parameter space and partial-wave analyses.

For the case of $\mathcal{N}=8$ supergravity a recent paper [25] analyzes the eikonal phase through $\mathcal{O}\left(G^{4}\right)$ using the twoand three-loop amplitudes from Refs. [17,26]. The same work [25] observes that the $\mathcal{N}=8$ scattering angle matches the angle found by ACV through $\mathcal{O}\left(G^{3}\right)$ [3], despite having different matter content. Indeed, as we show 
here, this is not an accident, but part of a general pattern. Our explicit calculations for the $\mathcal{O}\left(G^{3}\right)$ contributions to the classical scattering angle in $\mathcal{N} \geq 4$ and pure gravity give the identical result as the angle found by ACV, demonstrating its universality.

The classical limit of the amplitude.-We are interested in extracting the contributions to the conservative classical scattering angle from the two-loop four-point scattering amplitudes of Refs. [15-18]. Four-point scattering amplitudes depend on the kinematic invariants $s$ and $t=-q^{2}$, which in the center of mass frame correspond to the squared total energy and squared four-momentum transfer, respectively. We consider the amplitude in the physical region $s>0, t<0, u=-s-t<0$ (using a mostly minus sign convention for the metric), commonly known as the $s$ channel. The contributions in the amplitude relevant for the classical angle corresponds to the large angular momentum limit, which for massless particles is $J \sim \sqrt{s} b \gg 1$, where $b$ denotes the usual impact parameter. In the absence of any other kinematic scales such as masses in the momentumspace scattering amplitude, the classical limit is equivalent to the Regge or high-energy small-angle limit, $s / q^{2} \gg 1$. It is straightforward to argue that the singularity structure of massless scattering amplitudes implies that only even loop orders can give rise to classical contributions (see, e.g., Refs. [3,25] for a detailed argument). At one loop, in particular, this is directly tied to the fact that no term behaves as $1 / q$ which would be required to contribute to the classical deflection angle.

Following Ref. [3], we consider external graviton states. For simplicity we focus on the configuration where the incoming and outgoing gravitons in the $s$ channel have identical helicity; the situation where the incoming and outgoing gravitons have opposite helicity gives the same final classical scattering angle. We extract the classical scattering angle from the Regge limit of the renormalized scattering amplitudes, which take the following form,

$$
\begin{aligned}
\mathcal{M}^{(0)}\left(s, q^{2}\right) & =\mathcal{K} 8 \pi G s\left[\frac{s}{q^{2}}+1\right], \\
\mathcal{M}^{(1)}\left(s, q^{2}\right) & =\left(2 \pi G ^ { 2 } s ^ { 2 } r _ { \Gamma } ( \frac { \overline { \mu } ^ { 2 } } { q ^ { 2 } } ) ^ { \epsilon } \left[-\frac{2 \pi i}{\epsilon} \frac{s}{q^{2}}\right.\right. \\
\mathcal{M}^{(2)}\left(s, q^{2}\right) & =(00)=2 \mathcal{K} G^{3} s^{3} \frac{r_{\Gamma}^{2}}{\pi}\left(\frac{\bar{\mu}^{2}}{q^{2}}\right)^{2 \epsilon}\left[-\frac{2 \pi^{2}}{\epsilon^{2}} \frac{s}{q^{2}}\right. \\
& \left.-\frac{2 \pi i}{\epsilon^{2}}(2 L+2-i \pi)-\frac{2 \pi i}{\epsilon} F^{(1)}+F^{(2)}\right],
\end{aligned}
$$

where we dropped subdominant terms of $\mathcal{O}\left(q^{2} / s\right)$ in the loop amplitudes, and where $\mathcal{K}$ is a local factor depending on the external states, $\bar{\mu}^{2} \equiv 4 \pi e^{-\gamma_{E}} \mu^{2}$ is a rescaled renormalization scale and $r_{\Gamma} \equiv e^{\epsilon \gamma_{E}} \Gamma(1+\epsilon) \Gamma(1-\epsilon)^{2} / \Gamma(1-2 \epsilon)$. For convenience we introduced $L=\log \left(s / q^{2}\right)$, and the finite remainders $F^{(i)}$, which depend on the theory and are implicitly defined in Eq. (1). This result is given in the conventional dimensional regularization scheme, where all internal states and momenta are analytically continued into $D=4-2 \epsilon$ dimensions. For the purposes of this Letter, we only need $F^{(1)}$ to $\mathcal{O}(\epsilon)$ and $F^{(2)}$ to $\mathcal{O}\left(\epsilon^{0}\right)$. The two-loop infrared singular part is related to the square of the one-loop amplitude via $\left[\mathcal{M}^{(1)}\right]^{2} / 2 \mathcal{M}^{(0)}$ which follows from the fact that to all loop orders the infrared singularity is given by an exponential of the ratio of the one-loop and tree amplitudes [16,27].

The pure gravity one-loop amplitudes were originally computed in Ref. [28]. These were recomputed in an intermediate step [29] of the two-loop analysis of Ref. [30]. This is matched by the expressions in Ref. [18] that include the $\mathcal{O}(\epsilon)$ contributions. The latter contributions are needed when extracting the two-loop finite remainders in the presence of infrared singularities, with the result,

$$
\begin{aligned}
F_{\mathrm{GR}}^{(1)}= & 2 L^{2}+2 i \pi L+4 \pi^{2}-\frac{87}{10} L+\frac{841}{90} \\
& +\epsilon\left[-\frac{2}{3} L^{3}-\pi^{2} L+6 \zeta_{3}+\frac{47}{20} L^{2}-3 \pi^{2}-\frac{6913}{225} L\right. \\
& \left.+\frac{35597}{1200}+i \pi\left(-L^{2}+\frac{\pi^{2}}{3}+10 L+\frac{1957}{360}\right)\right],
\end{aligned}
$$

where $F_{\mathrm{GR}}^{(1)}$ is the pure gravity result for $F^{(1)}$ in Eq. (1). The $\mathcal{N} \geq 4$ supergravity amplitudes can be found in Ref. [17,28,31] in a scheme that preserves supersymmetry. For these cases, the Regge limit of the $\mathcal{O}\left(\epsilon^{0}\right)$ contributions to the finite remainders can be read off from Eq. (4.6) of Ref. [22].

Reference [18] provides the complete Einstein-gravity amplitude needed for our analysis, including subdivergence subtractions [29,30,32]. We note that these results pass highly nontrivial checks. The amplitude yields the expected IR pole structure [27] and the net ultraviolet poles cancel against the known counterterms [30,33]. Furthermore the amplitude only has the poles in the Mandelstam variables $s$, $t$, and $u$ dictated by factorization. The amplitudes have also been validated against results in the literature and independent computations. While not directly relevant for the classical scattering angle, the results of Ref. [18] also match the previously computed [29] identical-helicity amplitude (in an all outgoing momentum convention), corresponding to the case that both incoming gravitons flip helicity.

Starting from the full four-graviton two-loop amplitude in pure Einstein gravity [18], we extract the finite remainder in the Regge limit giving the result, 


$$
\begin{aligned}
F_{\mathrm{GR}}^{(2)}= & -2 \pi^{2} L^{2}+4 \pi^{2} L-\frac{\pi^{4}}{90}+\frac{13403 \pi^{2}}{675}-\frac{13049}{2160} \\
& +i \pi\left[\frac{4}{3} L^{3}-\frac{47}{10} L^{2}+\frac{26159}{450} L-20 \zeta_{3}\right. \\
& \left.+\frac{2621 \pi^{2}}{210}-\frac{11221}{375}\right] .
\end{aligned}
$$

The constant parts are scheme dependent and in any case they do not affect the scattering angle. A detailed discussion of scheme dependence and its effects on the final angle, in the context of IR regulators in $\mathcal{N}=8$ supergravity is found in Section VI of Ref. [25].

The two-loop amplitudes for $\mathcal{N} \geq 4$ supergravity are given in Ref. [17]. The $\mathcal{N}=8$ supergravity result is the simplest of these and was first given in Ref. [16] by combining the integrand of Ref. [15] with the integrals of Ref. [34]. Explicit results for the finite remainders in the Regge limit are found in Eqs. (4.13)-(4.16) of Ref. [22]. Note that the remainders in Ref. [22] are normalized with an extra factor of $q^{2} s$ relative to ours.

So far we have presented the classical scattering amplitudes in perturbation theory, which assumes $G s \ll 1$. Ultimately, we are interested in the limit $G s \gg 1$, with $G s / J \ll 1$ corresponding to the classical postMinkowskian expansion used in classical general relativity [35]. Implicitly this assumes that the relevant parts of the perturbative series have been resummed. Standard ways to do so use eikonal or partial wave methods which we utilize in the following.

Scattering angle from eikonal phase.-Following the usual procedure $[3,20,21,25]$, we obtain the eikonal phase by taking the transverse Fourier transform of the amplitude in the classical limit,

$$
-i\left(e^{i 2 \delta\left(s, b_{e}\right)}-1\right)=\int \frac{\mu^{2 \epsilon} d^{2-2 \epsilon} q}{(2 \pi)^{2-2 \epsilon}} e^{i \vec{q} \cdot \vec{b}_{e}} \frac{\mathcal{M}\left(s, q^{2}\right)}{2 s \mathcal{K}},
$$

where $\delta\left(s, b_{e}\right)$ is the eikonal phase, which we expand perturbatively in Newton's constant $\left(\delta=\delta^{(0)}+\right.$ $\left.\delta^{(1)}+\delta^{(2)}+\cdots\right), \vec{q}$ is the $(2-2 \epsilon)$-dimensional vector in the scattering plane such that $\vec{q}^{2}=q^{2}$ and $b_{e} \equiv\left|\vec{b}_{e}\right|$ is the eikonal impact parameter shown in Fig. 1. The basic formula needed for calculating the Fourier transform is given in Eq. (2.11) of Ref. [24].

The full phase shift is generically complex, and be readily obtained from Eqs. (1), (2), and (3). Its imaginary part at a given order captures inelastic (e.g., radiation) effects. Here we are only interested in the conservative part, as in Ref. [3] so we do not display it in the following and focus only on the elastic phase. However, these imaginary parts are needed to extract the elastic contributions at higher orders because of the exponentiation. The Fourier transform of polynomial terms corresponds to short-range

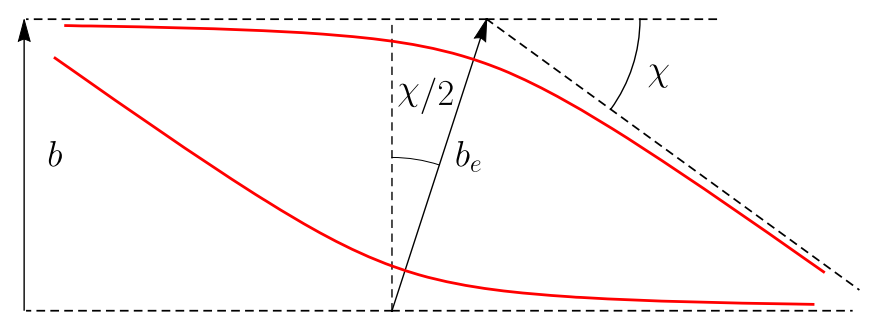

FIG. 1. The scattering configuration showing the impact parameter, $b$, eikonal impact parameter, $b_{e}$, and the scattering angle, $\chi$.

contact interactions, which are not relevant for the problem of long-range scattering.

The universal $\mathcal{O}(G)$ result for the eikonal phase extracted from the tree amplitude is

$$
\delta^{(0)}=\frac{G s}{2}\left(\bar{\mu}^{2} \tilde{b}_{e}^{2}\right)^{\epsilon}\left[-\frac{1}{\epsilon}-\epsilon \frac{\pi^{2}}{12}-\frac{1}{3} \epsilon^{2} \zeta_{3}+\mathcal{O}\left(\epsilon^{3}\right)\right],
$$

where we introduced $\tilde{b}_{e}=e^{\gamma_{E}} b_{e} / 2$ for convenience.

As explained above, the pieces relevant for the one-loop scattering angle are given by the real part of the nonanalytic part,

$$
\operatorname{Re} F^{(1)}=-\frac{\mathcal{N}-4}{2} L^{2}+c L+\cdots,
$$

where $\mathcal{N}$ denotes the amount of supersymmetry and $c$ is a constant that takes on the values $0,-1,-87 / 10$ for $\mathcal{N}>4$, $\mathcal{N}=4$ and pure gravity, respectively. The leading logarithms $\left(L^{2}\right)$ arise from backward-scattering diagrams [22] and the subleading logarithm $(L)$ from bubble integrals. We conclude that they are nonuniversal and depend on the specific theory. As mentioned above, the $\mathcal{O}\left(G^{2}\right)$ one-loop phase can contribute to the angle only at the quantum level, so this nonuniversality does not affect the classical scattering angle. These contributions, including the $\mathcal{O}(\epsilon)$ parts, are however crucial for extracting the $\mathcal{O}\left(G^{3}\right)$ classical pieces because of cross terms with infrared singularities.

The $\mathcal{O}\left(G^{2}\right)$ phase extracted from the one-loop amplitude is

$$
\begin{aligned}
\operatorname{Re} \delta^{(1)}= & \frac{2 G^{2} s}{\pi b_{e}^{2}}\left(\bar{\mu}^{2} \tilde{b}_{e}^{2}\right)^{2 \epsilon} \\
& \times\left[\frac{1}{\epsilon}-\frac{(\mathcal{N}-6)}{2} \log \left(s \tilde{b}_{e}^{2}\right)+\frac{c+2}{2}+\mathcal{O}(\epsilon)\right],
\end{aligned}
$$

where $c$ is the same theory-dependent constant appearing in Eq. (6). Additionally, there is an imaginary part at $\mathcal{O}(\epsilon)$, needed to obtain the real part of $\delta^{(2)}$, which is not displayed here but is readily obtained from the Fourier transform of the full amplitudes in Eqs. (1) and (2) as well from Refs. [28-30]. 
The relevant terms at two loops arise from the nonanalytic terms in the imaginary part of the remainder at one loop and from the real part at two loops

$$
\begin{aligned}
& \operatorname{Im} F^{(1)}=2 \pi L-\epsilon \pi L^{2}+\cdots, \\
& \operatorname{Re} F^{(2)}=-2 \pi^{2} L^{2}+4 \pi^{2} L+\cdots,
\end{aligned}
$$

where the dots indicate nonuniversal terms which do not contribute to the phase at $\mathcal{O}\left(\epsilon^{0}\right)$. This includes nonuniversal $\epsilon L$ terms in $\operatorname{Im} F^{(1)}$ that could naively contribute but ultimately cancels against the iteration $-2 i \delta^{(0)} \delta^{(1)}$ coming from expanding the exponential.

The $\mathcal{O}\left(G^{3}\right)$ terms in the phase can thus be extracted from the two-loop amplitude after subtracting the iteration from the leading and subleading phases in the exponential (4). The leading eikonal exponentiation also predicts a universal $\mathcal{O}(\epsilon)$ contribution to the two-loop amplitude which needs to be taken into account. [See the discussion in Ref. [25] near Eq. (3.7)]. We obtain the universal result,

$$
\operatorname{Re} \delta^{(2)}=\frac{2 G^{3} s^{2}}{b_{e}^{2}}\left(\bar{\mu}^{2} \tilde{b}_{e}^{2}\right)^{3 \epsilon}+\mathcal{O}(\epsilon),
$$

valid for $\mathcal{N} \geq 4$ supergravity as well as pure Einstein gravity. We are not displaying the imaginary parts since they are not universal and do not contribute to the conservative dynamics at this order.

The classical scattering angle is given in terms of the eikonal phase via the usual stationary-phase argument (see, e.g., [2]),

$$
\sin \frac{1}{2} \chi\left(s, b_{e}\right)=-\frac{2}{\sqrt{s}} \frac{\partial}{\partial b_{e}} \delta\left(s, b_{e}\right) .
$$

Applying this formula to Eq. (9), which holds for all theories evaluated here, we obtain the universal result

$$
\sin \frac{1}{2} \chi\left(s, b_{e}\right)=\frac{2 G \sqrt{s}}{b_{e}}+\frac{(2 G \sqrt{s})^{3}}{b_{e}^{3}},
$$

matching the ACV pure gravity angle given in Eq. (5.28) in Ref. [3], as well as the recently obtained angle in $\mathcal{N}=8$ supergravity [25]. The scheme dependence cancels, as expected. The result above is written in terms of the symmetric impact parameter, $\vec{b}_{e}$ which appears naturally in the eikonal formula. This points in the direction of the momentum transfer $\vec{q}$, while the more familiar impact parameter $\vec{b}$ is perpendicular to the incoming momenta, as shown in Fig. 1. (See also Ref. [23].) The relation between their magnitudes is $b=b_{e} \cos (\chi / 2)$. Rewriting the universal scattering angle in terms of the usual impact parameter $b$ gives,

$$
\sin \frac{1}{2} \chi(s, b)=\frac{2 G \sqrt{s}}{b}+\frac{1}{2} \frac{(2 G \sqrt{s})^{3}}{b^{3}} .
$$

We note that the quantum corrections to the scattering angle do not display a corresponding universality, analogous to previously observed nonuniversal spin dependence in quantum corrections [36].

Scattering angle from partial-wave expansion.Alternatively, we can extract the scattering angle from the partial-wave expansion of the amplitude (see, e.g., Ref. [12]). Here we note that the partial waves are given by

$a_{l}(s)=\frac{\left(16 \pi \mu^{2} / s\right)^{\epsilon}}{\Gamma(1-\epsilon)} \int_{-1}^{1} d x\left(1-x^{2}\right)^{-\epsilon} C_{l}^{\frac{1-2 \epsilon}{-2}}(x) \frac{\mathcal{M}(s, x)}{16 \pi \mathcal{K}}$,

where $x=\cos \chi=1+2 t / s$ and the $C_{l}^{(1-2 \epsilon) / 2}(x)$ are Gegenbauer polynomials (normalized to take unit value at $x=1$ ), which reduce to the more familiar Legendre polynomials when $\epsilon \rightarrow 0$.

If we ignore inelastic contributions, the partial waves can be parametrized in terms of phase shifts as

$$
a_{l}(s)=-i\left(e^{i 2 \delta_{l}(s)}-1\right),
$$

and once again a stationary-phase argument gives the scattering angle as

$$
\frac{1}{2} \chi(s, l)=-\frac{\partial \delta_{l}(s)}{\partial l} .
$$

Using this approach we find the phase shifts,

$$
\begin{aligned}
\delta_{l}^{(0)}(s)= & \frac{G s}{2}\left(\frac{\bar{\mu}^{2} \tilde{J}^{2}}{s}\right)^{\epsilon}\left[-\frac{1}{\epsilon}-\frac{1}{3 J^{2}}+\mathcal{O}\left(\epsilon, J^{-4}\right)\right], \\
\operatorname{Re} \delta_{l}^{(1)}(s)= & \frac{G^{2} s^{2}}{2 \pi J^{2}}\left(\frac{\bar{\mu}^{2} \tilde{J}^{2}}{s}\right)^{2 \epsilon} \\
& \times\left[\frac{1}{\epsilon}-\frac{(\mathcal{N}-6)}{2} \log \left(\tilde{J}^{2}\right)+\frac{c+2}{2}+\mathcal{O}\left(\epsilon, J^{-2}\right)\right], \\
\operatorname{Re} \delta_{l}^{(2)}(s)= & \frac{G^{3} s^{3}}{3 J^{2}}\left(\frac{\bar{\mu}^{2} \tilde{J}^{2}}{s}\right)^{3 \epsilon}+\mathcal{O}\left(\epsilon, J^{-4}\right),
\end{aligned}
$$

where $\tilde{J}^{2}=e^{2 \gamma_{E}} J^{2}$ and $J^{2}$ denotes the Casimir of the rotation group, i.e., $J^{2}: \equiv l(l+1-2 \epsilon)$, which has a well-defined classical limit. The classical deflection angle is then

$$
\frac{1}{2} \chi(s, J)=\frac{G s}{J}+\frac{2}{3} \frac{G^{3} s^{3}}{J^{3}},
$$

written in terms of the classical variables, or, equivalently, 


$$
\sin \frac{1}{2} \chi(s, J)=\frac{G s}{J}+\frac{1}{2} \frac{G^{3} s^{3}}{J^{3}} .
$$

Using the relation between the angular momentum and the impact parameters

$$
J=\frac{\sqrt{s}}{2} b=\frac{\sqrt{s}}{2} b_{e} \cos \frac{1}{2} \chi,
$$

we find that Eq. (18) reproduces Eqs. (11) and (12).

We can directly compare our results to Damour's conjectured angle given in Eq. (5.37) of Ref. [12],

$$
\sin \frac{1}{2} \chi^{D}(s, J)=\frac{G s}{J}-\frac{3}{4} \frac{G^{3} s^{3}}{J^{3}} .
$$

As noted in Ref. [12], this disagrees with the angle obtained by ACV, which is matched by Eq. (18). As emphasized by Damour [12], because the sign of the $G^{3}$ term in Eq. (20) is opposite to that of Eq. (18) the disagreement between the two formulas is robust.

Here we focused on the scattering of identical-helicity gravitons in the initial state. We have repeated the calculation for the case of opposite-helicity gravitons with the same results for the classical scattering angle. Furthermore, we expect the result to be identical for any massless external states. Indeed, for the supersymmetric cases that we analyzed, supersymmetry identities [37] relate graviton scattering to scattering of other massless states.

Conclusions.-By studying gravitational scattering amplitudes through $\mathcal{O}\left(G^{3}\right)$ in a variety of theories, we found the classical scattering angle to be independent of their matter content, thus demonstrating graviton dominance at a higher order than had been previously understood [2]. In addition, we confirmed that the classical scattering angle found by ACV [3] is indeed correct. The results of our calculation are, however, in conflict with Damour's recent conjecture [12].

There are a number of interesting directions to pursue. First and foremost, it would be desirable to systematically complete a proof of universality through $\mathcal{O}\left(G^{3}\right)$ for any massless gravitational theory. An obvious, if nontrivial, next step would be to check whether some form of universality remains at higher orders as well. It would also be important to understand the constraints that the high-energy behavior of scattering amplitudes imposes on classical binary black hole interactions [10]. The recent advances $[19,38]$ that make it possible to obtain the complete four-graviton twoloop amplitude of pure Einstein gravity [18] can be expected to lead to further advances, including for the important case of massive multiloop amplitudes relevant for the gravitational-wave two-body problem.

We thank Alessandra Buonanno, Clifford Cheung, Marcello Ciafaloni, Thibault Damour, Paolo Di Vecchia, David Kosower, Andrés Luna, Stephen Naculich, Radu
Roiban, Rodolfo Russo, Donal O'Connell, Chia-Hsien Shen, Mikhail Solon, Jan Steinhoff, Justin Vines, Zahra Zahraee, and especially Simon Caron-Huot and Gabriele Veneziano for many helpful comments and discussions. We thank the U.S. Department of Energy (DOE) for support under Grant No. DE-SC0009937. J. P. M. is supported by the U.S. Department of State through a Fulbright scholarship. M. S. R.'s work is funded by the German Research Foundation (DFG) within the Research Training Group GRK 2044. We are also grateful to the Mani L. Bhaumik Institute for Theoretical Physics for generous support.

Note added.-Recently, new calculations [39] confirm that terms in the 6th post-Newtonian order agree with the results of Refs. [6,7], but they are inconsistent with the conjecture of Ref. [12].

[1] P. D. D'Eath, Phys. Rev. D 18, 990 (1978); S. J. Kovacs and K. S. Thorne, Astrophys. J. 217, 252 (1977); 224, 62 (1978).

[2] D. Amati, M. Ciafaloni, and G. Veneziano, Phys. Lett. B 197, 81 (1987); G. 't Hooft, Phys. Lett. B 198, 61 (1987); D. Amati, M. Ciafaloni, and G. Veneziano, Int. J. Mod. Phys. A 03, 1615 (1988); I. J. Muzinich and M. Soldate, Phys. Rev. D 37, 359 (1988); S. B. Giddings, M. Schmidt-Sommerfeld, and J. R. Andersen, Phys. Rev. D 82, 104022 (2010).

[3] D. Amati, M. Ciafaloni, and G. Veneziano, Nucl. Phys. B347, 550 (1990).

[4] B. P. Abbott et al. (LIGO Scientific and Virgo Collaborations), Phys. Rev. Lett. 116, 061102 (2016); 119, 161101 (2017).

[5] Y. Iwasaki, Prog. Theor. Phys. 46, 1587 (1971); Y. Iwasaki, Lett. Nuovo Cimento 1, 783 (1971); 1, 783 (1971); H. Okamura, T. Ohta, T. Kimura, and K. Hiida, Prog. Theor. Phys. 50, 2066 (1973); S. N. Gupta and S. F. Radford, Phys. Rev. D 19, 1065 (1979); J. F. Donoghue, Phys. Rev. D 50, 3874 (1994); B. R. Holstein and J. F. Donoghue, Phys. Rev. Lett. 93, 201602 (2004); D. Neill and I. Z. Rothstein, Nucl. Phys. B877, 177 (2013); V. Vaidya, Phys. Rev. D 91, 024017 (2015); N. E. J. Bjerrum-Bohr, J. F. Donoghue, and P. Vanhove, J. High Energy Phys. 02 (2014) 111; N. E. J. Bjerrum-Bohr, P. H. Damgaard, G. Festuccia, L. Planté, and P. Vanhove, Phys. Rev. Lett. 121, 171601 (2018); C. Cheung, I. Z. Rothstein, and M. P. Solon, Phys. Rev. Lett. 121, 251101 (2018); D. A. Kosower, B. Maybee, and D. O’Connell, J. High Energy Phys. 02 (2019) 137.

[6] Z. Bern, C. Cheung, R. Roiban, C. H. Shen, M. P. Solon, and M. Zeng, Phys. Rev. Lett. 122, 201603 (2019).

[7] Z. Bern, C. Cheung, R. Roiban, C. H. Shen, M. P. Solon, and M. Zeng, J. High Energy Phys. 10 (2019) 206.

[8] Z. Bern, L. J. Dixon, D. C. Dunbar, and D. A. Kosower, Nucl. Phys. B425, 217 (1994); 435, 59 (1995); Z. Bern, L. J. Dixon, and D. A. Kosower, Nucl. Phys. B513, 3 (1998); R. Britto, F. Cachazo, and B. Feng, Nucl. Phys. B725, 275 (2005); Z. Bern, J. J. M. Carrasco, H. Johansson, and D. A. Kosower, Phys. Rev. D 76, 125020 (2007).

[9] H. Kawai, D. C. Lewellen, and S. H. H. Tye, Nucl. Phys. B269, 1 (1986); Z. Bern, L. J. Dixon, M. Perelstein, and J. S. 
Rozowsky, Nucl. Phys. B546, 423 (1999); Z. Bern, John Joseph M. Carrasco, and H. Johansson, Phys. Rev. D 78, 085011 (2008); Phys. Rev. Lett. 105, 061602 (2010); Z. Bern, J. J. Carrasco, M. Chiodaroli, H. Johansson, and R. Roiban, arXiv:1909.01358.

[10] T. Damour, Phys. Rev. D 97, 044038 (2018).

[11] A. Buonanno and T. Damour, Phys. Rev. D 59, 084006 (1999); A. Buonanno and T. Damour, Phys. Rev. D 62, 064015 (2000).

[12] T. Damour, arXiv:1912.02139v1.

[13] Y. Mino, M. Sasaki, and T. Tanaka, Phys. Rev. D 55, 3457 (1997); T. C. Quinn and R. M. Wald, Phys. Rev. D 56, 3381 (1997).

[14] A. Bellini, M. Ademollo, and M. Ciafaloni, Nucl. Phys. B393, 79 (1993).

[15] Z. Bern, L. J. Dixon, D. C. Dunbar, M. Perelstein, and J. S. Rozowsky, Nucl. Phys. B530, 401 (1998).

[16] S. G. Naculich, H. Nastase, and H. J. Schnitzer, Nucl. Phys. B805, 40 (2008); A. Brandhuber, P. Heslop, A. Nasti, B. Spence, and G. Travaglini, Nucl. Phys. B807, 290 (2009).

[17] C. Boucher-Veronneau and L. J. Dixon, J. High Energy Phys. 12 (2011) 046.

[18] S. Abreu, F. F. Cordero, H. Ita, M. Jaquier, B. Page, M. S. Ruf, and V. Sotnikov, arXiv:2002.12374.

[19] H. Ita, Phys. Rev. D 94, 116015 (2016); S. Abreu, F. F. Cordero, H. Ita, M. Jaquier, B. Page, and M. Zeng, Phys. Rev. Lett. 119, 142001 (2017).

[20] R. J. Glauber, in Lectures in Theoretical Physics, edited by W. E. Brittin and L. G. Dunham (Interscience Publishers, Inc., New York, 1959), Vol. I, p. 315.

[21] E. Laenen, G. Stavenga, and C. D. White, J. High Energy Phys. 03 (2009) 054; R. Akhoury, R. Saotome, and G. Sterman, arXiv:1308.5204; P. Di Vecchia, A. Luna, S. G. Naculich, R. Russo, G. Veneziano, and C. D. White, Phys. Lett. B 798, 134927 (2019); M. Kulaxizi, G. S. Ng, and A. Parnachev, J. High Energy Phys. 10 (2019) 107.

[22] S. Melville, S. G. Naculich, H. J. Schnitzer, and C. D. White, Phys. Rev. D 89, 025009 (2014).

[23] M. Ciafaloni and D. Colferai, J. High Energy Phys. 10 (2014) 085.

[24] A. Koemans Collado, P. Di Vecchia, and R. Russo, Phys. Rev. D 100, 066028 (2019).

[25] P. Di Vecchia, S. G. Naculich, R. Russo, G. Veneziano, and C. D. White, arXiv:1911.11716.

[26] J. M. Henn and B. Mistlberger, J. High Energy Phys. 05 (2019) 023.
[27] S. Weinberg, Phys. Rev. 140, B516 (1965); S. G. Naculich and H. J. Schnitzer, J. High Energy Phys. 05 (2011) 087.

[28] D. C. Dunbar and P. S. Norridge, Nucl. Phys. B433, 181 (1995).

[29] Z. Bern, C. Cheung, H.-H. Chi, S. Davies, L. Dixon, and J. Nohle, private communication.

[30] Z. Bern, C. Cheung, H. H. Chi, S. Davies, L. Dixon, and J. Nohle, Phys. Rev. Lett. 115, 211301 (2015); Z. Bern, H. H. Chi, L. Dixon, and A. Edison, Phys. Rev. D 95, 046013 (2017).

[31] Z. Bern, C. Boucher-Veronneau, and H. Johansson, Phys. Rev. D 84, 105035 (2011).

[32] G. 't Hooft and M. J. G. Veltman, Ann. Inst. Henri Poincare A 20, 69 (1974).

[33] M. H. Goroff and A. Sagnotti, Nucl. Phys. B266, 709 (1986); A. E. M. van de Ven, Nucl. Phys. B378, 309 (1992).

[34] V. A. Smirnov, Phys. Lett. B 460, 397 (1999); J. B. Tausk, Phys. Lett. B 469, 225 (1999).

[35] B. Bertotti, Nuovo Cimento 4, 898 (1956); R. P. Kerr, Nuovo Cimento (1955-1965) 13, 469 (1959); B. Bertotti and J. F. Plebański, Ann. Phys. (N.Y.) 11, 169 (1960); M. Portilla, J. Phys. A 12, 1075 (1979); K. Westpfahl and M. Goller, Lett. Nuovo Cimento 26, 573 (1979); M. Portilla, J. Phys. A 13, 3677 (1980); L. Bel, T. Damour, N. Deruelle, J. Ibanez, and J. Martin, Gen. Relativ. Gravit. 13, 963 (1981); K. Westpfahl, Fortschr. Phys. 33, 417 (1985); T. Ledvinka, G. Schafer, and J. Bicak, Phys. Rev. Lett. 100, 251101 (2008); T. Damour, Phys. Rev. D 94, 104015 (2016).

[36] N. E. J. Bjerrum-Bohr, J. F. Donoghue, B. R. Holstein, and L. Planté, and P. Vanhove, Phys. Rev. Lett. 114, 061301 (2015); N. E. J. Bjerrum-Bohr, J. F. Donoghue, B. R. Holstein, L. Planté, and P. Vanhove, J. High Energy Phys. 11 (2016) 117; D. Bai and Y. Huang, Phys. Rev. D 95, 064045 (2017); H. H. Chi, Phys. Rev. D 99, 126008 (2019).

[37] M. T. Grisaru, H. N. Pendleton, and P. van Nieuwenhuizen, Phys. Rev. D 15, 996 (1977); M. T. Grisaru and H. N. Pendleton, Nucl. Phys. B124, 81 (1977).

[38] S. Abreu, F. F. Cordero, H. Ita, B. Page, and M. Zeng, Phys. Rev. D 97, 116014 (2018); S. Abreu, J. Dormans, F. F. Cordero, H. Ita, and B. Page, Phys. Rev. Lett. 122, 082002 (2019).

[39] J. Blümlein, A. Maier, P. Marquard, and G. Schäfer, arXiv:2003.07145; C. Cheung and M. P. Solon, arXiv:2003.08351; D. Bini, T. Damour, and A. Geralico, arXiv:2003.11891. 